\title{
Short Communication \\ Overweight Risk and Food Habits in Portuguese Pre-school Children
}

\author{
A.M. Machado-Rodrigues ${ }^{1,2, *}$, R.A. Fernandes ${ }^{3}$, Maria-Raquel Silva ${ }^{5}$, A. Gama ${ }^{1,6}$, I. Mourão ${ }^{4}$, H. Nogueira ${ }^{1}$, \\ V. Rosado-Marques ${ }^{1,7}$, C. Padez ${ }^{1}$ \\ ${ }^{1}$ Research Centre for Anthropology and Health, University of Coimbra, Portugal \\ ${ }^{2}$ High School of Education, Polytechnic Institute of Viseu, Viseu, Portugal \\ ${ }^{3}$ Institute of Biosciences and Technology, São Paulo State University - UNESP, Brazil \\ ${ }^{4}$ Research Centre in Health and Human Development, University of Tras-os-Montes and Alto Douro, Portugal \\ ${ }^{5}$ Fernando Pessoa University, Porto, Portugal \\ ${ }^{6}$ Faculdade de Ciências, Universidade de Lisboa, Portugal \\ ${ }^{7}$ Faculdade de Motricidade Humana, Universidade de Lisboa, Portugal
}

\section{ARTICLE INFO}

Article History

Received 8 July 2017

Accepted 18 October 2017

Keywords

Overweight

public health

diet

pre-school children

\section{ABSTRACT}

The relationship between dietary intake and overweight-risk was assessed in 4349 children aged 3-5 years. Eating and sedentary behaviours were assessed by questionnaire. Logistic regressions were used. Children who consumed daily soft-drinks were 1.52 times more likely to be obese, and $72 \%$ more likely to be classified as overweight children.

\section{INTRODUCTION}

Childhood obesity is an important public health issue with a multifactorial nature. The consumption of sweetened beverages, such as regular soft drinks, and sweet snacks and candy are potential contributors to the increased of the prevalence of obesity $[1,2]$. Although several risk factors for consumption of the afore-mentioned nutritional items have been identified among school-aged children and adolescents [1,3], limited research has been conducted among pre-school children.

In fact, epidemiological studies that associate food intake and obesity are generally carried out in populations exposed to a Western dietary pattern of intake, characterized by high intake of fat, refined grains, red meat, sugary desserts and high-sugar drinks $[4,5]$. None of the above-mentioned studies were carried out in a Mediterranean country. Therefore, this information achieves greater insight since children from Southern countries of Europe have the highest prevalence of overweight/obesity.

In context of the preceding trends, the present study aimed to examine the relationships between dietary intake and overweight risk in a sample of Portuguese pre-school children.

${ }^{*}$ Corresponding author.Email: rodriguesari@hotmail.com

\section{MATERIAL AND METHODS}

\subsection{Participants}

The Portuguese Prevalence Study of Obesity in Childhood (PPSOC) was a random cross-sectional survey conducted in Portugal. Details on sampling and response rates can be found elsewhere [6]. In the present analyses, 4.349 pre-school children aged 3-5 were included. Ethical approval for PPSOC was given by the Portuguese Commission for Data Protection. Prior to data collection, informed written assent was obtained from participants and informed consent was obtained from parents or guardians.

\subsection{Anthropometry}

Height and weight were measured by two trained technicians at school in the morning, using a portable Seca 217 standiometer and portable Seca 770 scales to the nearest $0.1 \mathrm{~cm}$ and $0.1 \mathrm{~kg}$, respectively, with participants in t-shirt and shorts. Body mass index $\left(\mathrm{BMI}, \mathrm{kg} / \mathrm{m}^{2}\right)$ was calculated and categorized using age and sex-adjusted cut-off points [7]. The sample was divided into two weight-status groups, normal weight and overweight/obese. 


\subsection{Food Intake}

The children's dietary intake was measured using a semi-quantitative Food Frequency Questionnaire (FFQ). Each data set contained one FFQ regarding children's food intake and one sociodemographic questionnaire, both of which were asked to be filled out by their parents or tutors (the major responsible for their daily food intake).

Dietary intake was recorded using a semi-quantitative FFQ of the previous 12 months, comprising eighty-two food items or beverage categories and a frequency section with nine possible responses ranging from never to six or more times/day, developed by Lopes and colleagues [8,9] and adapted by inclusion of a variety of typical Portuguese food items for children.

\subsection{Sedentary Behaviour (SB)}

Screen time was the indicator of SB and was based on TV viewing. The amount of time spent viewing TV was determined from the proxy-report instrument as in other epidemiological studies of children $[10,11]$ and was expressed as $\mathrm{min} /$ day.

\subsection{Parental Education}

Educational background of fathers and mothers were used as a proxy for socio-economic status. It was based on the Portuguese Educational System [(1) 9 years or less - sub-secondary; (2) 10-12 years - secondary, and (3) higher education]. The three educational levels were defined as, respectively: 1 = Low Education; 2 = Middle Education and 3 = High Education. Similar procedures have used in the Portuguese context [12].

\subsection{Statistical Analysis}

Prior to analysis, normality of distribution of the afore-mentioned variables was verified using the Kolmogorov-Smirnov test.

Associations between weight status and the dietary intake assessed items, controlling for potentially confounding effects of chronological age, sex, time spent in sedentary behaviours (i.e. TV viewing) and, father and mother education were estimated using logistic regression analysis. In the minimally adjusted model (Model 1), each food item was the sole predictor of the child overweight/obesity. Chronological age and sex were subsequently added as potential confounders (Model 2). In Model 3, time spend sedentary (i.e. TV viewing) was added as a potential confounder. Finally, educational level of each parent was added as potential confounding factors (Model 4). The complex samples generalised linear models (CSGLM) procedure to produce results with robust standard errors that take into account clustering of participants by school were used. Significance was set at 5\%, and SPSS 15.0 (SPSS Inc., Chicago, Illinois, USA) was used.

\section{RESULTS}

Characteristics of the sample stratified by sex are summarized in Table 1. Based on the BMI, weight status of the sample was as follows: about $73 \%$ of girls were categorized as normal weight and
Table $1 \mid$ Descriptive data of Portuguese children aged 3-5 yrs

\begin{tabular}{|c|c|c|}
\hline & $\begin{array}{c}\text { Males } \\
(\mathrm{n}=\mathbf{2 2 1 0})\end{array}$ & $\begin{array}{l}\text { Females } \\
(n=2139)\end{array}$ \\
\hline Age, mean (Standard deviation) & $4.3(0.7)$ & $4.4(0.7)$ \\
\hline \multicolumn{3}{|l|}{ Weight status } \\
\hline Normal weight (\%) & $81.9(1809)$ & $73.1(1564)$ \\
\hline Overweight/obesity (\%) & $18.1(401)$ & $26.9(575)$ \\
\hline \multicolumn{3}{|l|}{ Screen time, daily (\%) } \\
\hline$<2 \mathrm{~h}$ & $74.0(1603)$ & $73.2(1566)$ \\
\hline$\geq 2 \mathrm{~h}$ & $26.0(607)$ & $26.8(573)$ \\
\hline \multicolumn{3}{|l|}{ Paternal education (\%) } \\
\hline Low & $41.9(925)$ & $42.0(891)$ \\
\hline Medium & $29.8(658)$ & $30.5(655)$ \\
\hline High & $28.3(627)$ & $27.5(594)$ \\
\hline \multicolumn{3}{|l|}{ Maternal education (\%) } \\
\hline Low & $37.0(817)$ & $39.7(848)$ \\
\hline Medium & $22.2(492)$ & $19.8(426)$ \\
\hline High & $40.8(901)$ & $40.5(865)$ \\
\hline \multicolumn{3}{|l|}{ Eating soup in a daily basis } \\
\hline Yes & $34.2(752)$ & $34.3(733)$ \\
\hline No & $65.8(1458)$ & $65.7(1406)$ \\
\hline \multicolumn{3}{|l|}{ Eating vegetables in a daily basis } \\
\hline Yes & $25.6(572)$ & $26.0(563)$ \\
\hline No & $74.4(1638)$ & $74.0(1576)$ \\
\hline \multicolumn{3}{|l|}{ Daily soft drinks } \\
\hline Yes & $4.3(96)$ & $4.3(91)$ \\
\hline No & $95.7(2214)$ & $95.7(2048)$ \\
\hline \multicolumn{3}{|l|}{ Eating chocolate in a daily basis } \\
\hline Yes & $9.9(213)$ & $9.3(212)$ \\
\hline No & $90.1(1997)$ & $90.7(1927)$ \\
\hline \multicolumn{3}{|l|}{ Breakfast at home } \\
\hline Yes & $97.3(2146)$ & $97.4(2079)$ \\
\hline No & $2.7(64)$ & $2.6(60)$ \\
\hline
\end{tabular}

$27 \%$ as overweight; corresponding values for boys were $82 \%, 18 \%$, respectively. About $36 \%$ of both boys and girls eat soup daily, and the just $24 \%$ of those children eat vegetables in a daily basis.

Associations between weight status and the dietary intake assessed items, controlling for potentially confounding effects of chronological age, sex, time spent in sedentary behaviours (i.e. TV viewing) and, father and mother education are presented in Table 2. After controlling for the afore-mentioned confounding effects, children who consumed soft drinks in a daily basis were 1.52 times more likely to be classified as overweight /obese than their peers without regular consumption of those beverages. The final regression model also showed that children who consumed chocolate regularly were $72 \%$ more likely to be classified as overweight/obese children.

\section{DISCUSSION}

Systematic evaluation of the independent contributions of dietary intake items to overweight/obesity risk in Portuguese children is lacking, particularly at the pre-school ages where data are quite difficult to collect. This is the first epidemiological study with a representative sample of pre-school children from a Mediterranean country which indicated a positive relationship between the overweight/obesity risk and soft drinks daily consumption in children aged 3-5 years. That association was not altered after adjustment for several potential confounding factors and was consistent with other studies [13] performed in children showing that greater 
Table 2 The association between diet habits and overweight/obesity controlling for biological and social variables in children aged 3-5 yrs

\begin{tabular}{|c|c|c|c|c|c|c|}
\hline \multirow{2}{*}{ Diet habits } & \multirow{2}{*}{ Model $^{\mathrm{a}}$} & \multicolumn{5}{|c|}{ Overweight/Obesity } \\
\hline & & $B$ & S.E. & $e^{B}$ & 95\% C.I. & $\mathbf{p}$ \\
\hline \multirow[t]{4}{*}{ Soup } & 1 & 0.45 & 0.09 & 1.05 & $0.88-1.25$ & 0.61 \\
\hline & 2 & 0.06 & 0.09 & 1.65 & $1.11-2.45$ & 0.48 \\
\hline & 3 & 0.06 & 0.09 & 1.07 & $0.89-1.27$ & 0.49 \\
\hline & 4 & 0.07 & 0.09 & 1.08 & $0.90-1.28$ & 0.41 \\
\hline \multirow[t]{4}{*}{ Vegetables } & 1 & 0.11 & 0.10 & 1.12 & $0.92-1.35$ & 0.26 \\
\hline & 2 & 0.11 & 0.10 & 1.12 & $0.92-1.35$ & 0.26 \\
\hline & 3 & 0.10 & 0.10 & 1.11 & $0.92-1.34$ & 0.29 \\
\hline & 4 & 0.08 & 0.10 & 1.08 & $0.90-1.31$ & 0.41 \\
\hline \multirow[t]{4}{*}{ Soft drinks } & 1 & 0.50 & 0.20 & 1.65 & $1.12-2.44$ & 0.01 \\
\hline & 2 & 0.50 & 0.20 & 1.65 & $1.11-2.45$ & 0.01 \\
\hline & 3 & 0.50 & 0.20 & 1.64 & $1.11-2.44$ & 0.01 \\
\hline & 4 & 0.42 & 0.20 & 1.52 & $1.02-2.25$ & 0.04 \\
\hline \multirow[t]{4}{*}{ Chocolates } & 1 & 0.33 & 0.15 & 0.72 & $0.53-0.97$ & 0.03 \\
\hline & 2 & 0.31 & 0.16 & 0.73 & $0.54-0.99$ & 0.04 \\
\hline & 3 & 0.32 & 0.16 & 0.72 & $0.53-0.98$ & 0.04 \\
\hline & 4 & 0.32 & 0.16 & 0.72 & $0.53-0.98$ & 0.04 \\
\hline \multirow{4}{*}{ Breakfast } & 1 & 0.40 & 0.25 & 1.49 & $0.92-2.41$ & 0.10 \\
\hline & 2 & 0.41 & 0.25 & 1.51 & $0.93-2.44$ & 0.10 \\
\hline & 3 & 0.40 & 0.25 & 1.50 & $0.92-2.43$ & 0.10 \\
\hline & 4 & 0.36 & 0.25 & 1.44 & $0.88-2.34$ & 0.15 \\
\hline
\end{tabular}

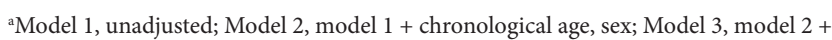
TV viewing; Model 4, model $3+$ paternal education, and maternal education.

intake of sweet beverages daily was associated with a worse weight profile in children.

In fact, the finding that especially sugar-rich foods and beverages contributed more to the energy intake is of concern. The majority of epidemiological studies clearly indicate that regular consumption of soft drinks may lead to weight gain and substantially increase the risk of developing chronic diseases [14]. In addition, an high intake of added sugars may increase the risk of a nutritionally inadequate diet [15] and is found to be positively associated with multiple measures known to increase CVD risk in youth. Of note, the present study revealed a positively association between BMI and the daily consumption of chocolate in 3-5 years-old children. This is of concern in the prevention of overweight in children because these dietary habits may promote positive energy balance, thereby increasing the risk of becoming overweight and obese.

On the other hand, epidemiological recent studies have also emphasised that children residing in neighbourhoods with greater access to fast foods and lower access to fruits and vegetables may be at higher risk for developing obesity during pre-school years [4]. Indeed, more than $20 \%$ of the children of the present study are overweight/obese, and therefore this high value supports the urgency of a nutritional intervention for pre-school Portuguese children, namely using education programmes in school, parents' education and control of the contents of school vending machines.

The major strengths of this study are the large sample of preschoolers and the variety of studied variables of those Southern European participants with high rates of overweight. On the other hand, limitations should be also recognized; firstly, this study has a cross-sectional design and, therefore, it is not possible to infer casual relationships. In addition, since data collection was performed by their parents, it may be associated with bias due to lack of parental knowledge of children's eating patterns away from home (i.e. at school).

\section{CONCLUSION}

Regular sweet beverages and chocolate intake were independently and positively associated with the overweight/obesity in pre-school children. These findings may help health professionals to advocate for policies that reduce sweet-food to improve healthy dietary.

\section{AUTHORS' CONTRIBUTION}

AMR, RF and CP participated in the design of the study and in its coordination and helped to draft the manuscript; AMR, AG, IM, HN, VRM and CP participated in the nutritional protocols, anthropometric measurements, data management and performed the statistical analysis. All authors revised the manuscript critically (eg. intellectual content analysis), and approved the final version of the present manuscript.

\section{DECLARATION OF INTEREST}

The authors report no conflicts of interest. The authors alone are responsible for the content and writing of the paper.

\section{ACKNOWLEDGMENT}

This research was partially supported by Fundação para a Ciência e a Tecnologia [FCOMP-01-0124-FEDER-007483].

\section{REFERENCES}

[1] Grimes CA, Wright JD, Liu K, Nowson CA, Loria CM. Dietary sodium intake is associated with total fluid and sugar-sweetened beverage consumption in US children and adolescents aged 2-18 y: NHANES 2005-2008. Am J Clin Nutr 2013;98;189-96.

[2] Malik VS, Schulze MB, Hu FB. Intake of sugar-sweetened beverages and weight gain: a systematic review. Am J Clin Nutr $2006 ; 84 ; 274-88$

[3] Valente H, Teixeira V, Padrao P, Bessa M, Cordeiro T, Moreira A, et al. Sugar-sweetened beverage intake and overweight in children from a Mediterranean country. Public Health Nutr 2011;14;127-32.

[4] Kepper M, Tseng TS, Volaufova J, Scribner R, Nuss H, Sothern M. Pre-school obesity is inversely associated with vegetable intake, grocery stores and outdoor play. Pediatr Obes 2016;11;e6-e8.

[5] Dubois L, Farmer A, Girard M, Peterson K. Social factors and television use during meals and snacks is associated with higher BMI among pre-school children. Public Health Nutr $2008 ; 11 ; 1267-79$.

[6] Machado-Rodrigues AM, Santana A, Gama A, Mourao I, Nogueira H, Rosado V, et al. Parental perceptions of neighborhood environments, BMI, and active behaviors in girls aged 7-9 years. Am J Hum Biol 2014;26;670-5. 
[7] Cole TJ, Bellizzi MC, Flegal KM, Dietz WH. Establishing a standard definition for child overweight and obesity worldwide: international survey. BMJ 2000;320;1240-3.

[8] Lopes C, Aro A, Azevedo A, Ramos E, Barros H. Intake and adipose tissue composition of fatty acids and risk of myocardial infarction in a male Portuguese community sample. J Am Diet Assoc 2007;107;276-86.

[9] Lopes C. Reproducibility and validation of a semiquantitative food-frequency questionnaire. In: Diet and acute myocardial infarction: a population-based casecontrol study. Porto: University of Porto, 2000. p. 79-115.

[10] Machado-Rodrigues AM, Leite N, Coelho-e-Silva MJ, Enes F, Fernandes R, Mascarenhas LP, et al. Metabolic risk and television time in adolescent females. Int J Public Health 2015;60;157-65.

[11] Machado-Rodrigues AM, Santana A, Gama A, Mourao I, Nogueira H, Rosado V, et al. Active commuting and its associations with blood pressure and adiposity markers in children. Prev Med 2014;69;132-4.

[12] Machado-Rodrigues AM, Coelho ESMJ, Mota J, Padez C, Martins RA, Cumming SP, et al. Urban-rural contrasts in fitness, physical activity, and sedentary behaviour in adolescents. Health Promot Int 2014;29;118-29.

[13] Dubuisson C, Lioret S, Dufour A, Calamassi-Tran G, Volatier JL, Lafay L, et al. Socio-economic and demographic variations in school lunch participation of French children aged 3-17 years. Public Health Nutr 2011;14;227-38.

[14] Hu FB, Malik VS. Sugar-sweetened beverages and risk of obesity and type 2 diabetes: epidemiologic evidence. Physiol Behav $2010 ; 100 ; 47-54$.

[15] Molgaard C, Andersen NL, Barkholt V, Grunnet N, Hermansen $\mathrm{K}$, Nyvad B, et al. The impact of sugar on health. Ugeskr Laeger 2003;165;4207-10. 